\title{
Rapid tests for the detection of the Mycobacterium abscessus subsp. bolletii strain responsible for an epidemic of surgical-site infections in Brazil
}

\author{
Cristianne Kayoko Matsumoto ${ }^{1 /}$, Erica Chimara ${ }^{2}$, Jesus Pais Ramos ${ }^{3}$, Carlos Eduardo Dias Campos ${ }^{3}$, \\ Paulo Cesar de Souza Caldas ${ }^{3}$, Karla Valeria Batista Lima ${ }^{4}$, Maria Luiza Lopes ${ }^{4}$, \\ Rafael Silva Duarte ${ }^{5}$, Sylvia Cardoso Leão ${ }^{1}$ \\ 1'Departamento de Microbiologia, Imunologia e Parasitologia, Escola Paulista de Medicina, Universidade Federal de São Paulo, \\ São Paulo, SP, Brasil ${ }^{2}$ Núcleo de Tuberculose e Micobacterioses, Instituto Adolfo Lutz, São Paulo, SP, Brasil \\ ${ }^{3}$ Centro de Referência Professor Hélio Fraga, Escola Nacional de Saúde Pública-Fiocruz, Rio de Janeiro, RJ, Brasil \\ ${ }^{4}$ Instituto Evandro Chagas, Belém, PA, Brasil ${ }^{5}$ nstituto de Microbiologia Professor Paulo de Góes, \\ Universidade Federal do Rio de Janeiro, Rio de Janeiro, RJ, Brasil
}

A single strain of Mycobacterium abscessus subsp. bolletii, characterised by a particular rpoB sequevar and two highly related pulsed field gel electrophoresis patterns has been responsible for a nationwide outbreak of surgical infections in Brazil since 2004. In this study, we developed molecular tests based on polymerase chain reaction restriction-enzyme analysis (PRA) and sequencing for the rapid identification of this strain. Sequences of 15 $D N A$ regions conserved in mycobacteria were retrieved from GenBank or sequenced and analysed in silico. Single nucleotide polymorphisms specific to the epidemic strain and located in enzyme recognition sites were detected in $\mathrm{rpoB}$, the 3' region of the $16 \mathrm{~S} r \mathrm{DNA}$ and gyrB. The three tests that were developed, i.e., PRA-rpoB, PRA-16S and gyrB sequence analysis, showed $100 \%, 100 \%$ and $92.31 \%$ sensitivity and $93.06 \%, 90.28 \%$ and $100 \%$ specificity, respectively, for the discrimination of the surgical strain from other $\mathrm{M}$. abscessus subsp. bolletii isolates, including 116 isolates from 95 patients, one environmental isolate and two type strains. The results of the three tests were stable, as shown by results obtained for different isolates from the same patient. In conclusion, due to the clinical and epidemiological importance of this strain, these tests could be implemented in reference laboratories for the rapid preliminary diagnosis and epidemiological surveillance of this epidemic strain.

Key words: Mycobacterium abscessus - epidemic - rapid test - rpoB - epidemiological surveillance

More than 2,000 cases of patients showing surgicalsite infections caused by rapidly growing mycobacteria (RGM) were reported to the Brazilian National Health Surveillance Agency (ANVISA) between 2003-2008. This was a sudden increase relative to previous years with only 13 cases reported between 2000-2003 (ANVISA 2009). Most of the infections were related to laparoscopic or arthroscopic surgeries and were caused by RGM with a Mycobacterium abscessus type 2 polymerase chain reaction (PCR) restriction-enzyme analysis (PRA)- $h s p 65$ pattern (BstE II: 235 and 210 bp; Hae III: 200, 70, 60 and $50 \mathrm{bp}$ ) (Devallois et al. 1997). The identification of these isolates was confirmed by rpoB sequencing (Adékambi et al. 2003). All of the obtained sequences were highly similar to the rpoB sequence of Mycobacterium massiliense CCUG 48898 (GenBank accession AY593981.2) and had two base substitutions (GenBank accession EU117207)

Financial support: CNPq (475238/2008-7)

CKM was the recipient of a fellowship from FAPESP (08/01451-8).

+ Corresponding author: criskmatsumoto@yahoo.com.br

Received 26 December 2011

Accepted 10 May 2012
(Leão et al. 2010). A total of 152 isolates obtained from surgical patients in different states belonged to a single strain and showed two closely related pulsed field gel electrophoresis (PFGE) patterns with a one-band difference when using either DraI or AseI restriction enzymes (Cardoso et al. 2008, Viana-Niero et al. 2008, Duarte et al. 2009, Leão et al. 2010). This strain was classified as $M$. abscessus subsp. bolletii according to the revised taxonomic status of the members of the Mycobacterium chelonae-M. abscessus group (Leão et al. 2009, 2011). One representative isolate from this strain was deposited in the collection of the National Institute for Quality and Health Control (INCQS), Rio de Janeiro (RJ), Brazil, under the accession INCQS 00594.

The epidemiological importance of this strain, which is herein referred to as the epidemic strain, is attributed to the extensive geographic and temporal occurrence of the epidemic of surgical-site infections (ANVISA 2011). Additionally, this strain was isolated from sputum, bronchoalveolar lavage fluid (BAL) and urine from patients unrelated to the outbreak (Matsumoto et al. 2011). The reports of RGM infections related to surgical procedures diminished significantly after 2008, although in 2010 additional surgical patients infected by the same strain were reported in the states of Amazonas (AM) and Rio Grande do Sul (RS), demonstrating that these infections may become endemic to Brazil. 
Currently, the identification of this particular strain is based on $r p o B$ sequencing and typing by PFGE. We have recently demonstrated that PFGE is a valid typing method for M. abscessus subsp. abscessus (Sampaio et al. 2006) and M. abscessus subsp. bolletii (Matsumoto et al. 2011). Sequencing and PFGE are not available in most public laboratories in Brazil and the epidemiological surveillance of this outbreak has suffered from the lack of a simple and rapid test to screen M. abscessus subsp. bolletii isolates, particularly when these isolates are derived from patients submitted to invasive procedures, such as laparoscopic, arthroscopic and plastic surgeries.

The objective of this study was to develop rapid tests for the specific identification of the epidemic strain among RGM isolates showing the M. abscessus type 2 PRA-hsp65 pattern.

\section{SUBJECTS, MATERIALS AND METHODS}

Mycobacterial isolates - A total of 116 mycobacterial isolates showing the M. abscessus type 2 PRA- $h s p 65$ pattern were included in this study. Isolates were classified as belonging to the epidemic strain when they showed one of the two PFGE patterns that were previously defined as being characteristic of this strain (Leão et al. 2010). Isolates showing highly similar PFGE patterns (differing from the epidemic strain patterns by up to three bands) corresponded to subtypes of the epidemic strain, according to van Belkum et al. (2007), and were categorised as genetically related (Matsumoto et al. 2011).

The type strains $M$. bolletii CCUG 50184 and $M$. massiliense CCUG 48898, both of which were reclassified as M. abscessus subsp. bolletii by Leão et al. (2011), and 55 clinical isolates from our collection were used in evaluation phases Ia and Ib (described in the Results section) (Table I). Only one isolate per patient was included. These isolates had been previously characterised by rрoB gene sequencing, PFGE-DraI and PFGE-AseI (Viana-Niero et al. 2008, da Costa et al. 2009, Duarte et al. 2009, Leão et al. 2010, Matsumoto et al. 2011).

Twenty coded isolates from 15 patients from the Institute of Microbiology Professor Paulo de Góes (Federal University of Rio de Janeiro) were sent to our laboratory (Mycobacteria Laboratory, Federal University of São Paulo) and used for the evaluation of the PRA-rpoB in evaluation phase IIa (Fig. 1).

Thirty-nine isolates from 26 patients, received between January 2009-May 2010 at the National Reference Laboratory Professor Hélio Fraga (CRPHF), RJ, were analysed locally by PRA-rpoB in evaluation phase IIb (Fig. 2).

The 59 isolates used in phases IIa and IIb were later characterised by $g y r B$ and $r p o B$ sequencing, PRA-16S and PFGE-DraI.

PFGE typing - PFGE typing was performed as described in previous publications from our group (Leão et al. 2010, Matsumoto et al. 2011). PFGE patterns were analysed using the BioNumerics v. 5.1 program (Applied Maths, Sint-Martens-Laten, Belgium).

$P C R$ and DNA sequencing - The primers used for the PCR amplification and sequencing of rpoB, the 3 ' region of the $16 \mathrm{~S}$ rDNA and gyrB genes are listed in Table II. The rров amplification was performed using primers $\mathrm{MycoF}$ and MycoR, as described by Adékambi et al. (2003). Two primers described in previous publications (Adékambi \& Drancourt 2004, Gomila et al. 2007) were used for the amplification and sequencing of the 3' region of the $16 \mathrm{~S}$ rDNA. The $\operatorname{gyr} B$ amplification primers were designed based on the sequence of the M. abscessus ATCC 19977 genome (GenBank accession NC_010397).

PCR products were purified by gel filtration (HiYield Gel/PCR DNA Extraction kit - RBC Biosciences Corp, Taipei, Taiwan). Dideoxy sequencing was performed using a BigDye ${ }^{\circledR}$ Terminator v3.1 Cycle Sequencing Kit (Applied Biosystems, Foster City, CA, USA) and an ABI PRISM 3100 DNA Analyzer (Applied Biosystems) according to the manufacturer's instructions.

The rpoB sequences from INCQS 00594, the $M$. massiliense and $M$. bolletti type strains, as well as the $16 \mathrm{~S}$ rDNA sequences from M. massiliense and M. bolletii, were obtained from GenBank (EU117207, AY593981.2, AY859692, AY593980 and AY859681).

DNA sequence analysis - Sequences were aligned using the CLUSTALW Multiple Alignment program (Thompson et al. 1994) included in the BioEdit v. 7.0.5.3 package (Hall 1999). Single nucleotide polymorphisms (SNPs) present exclusively in the INCQS 00594 sequences were identified. For the development of the PRA tests, SNPs located in enzyme recognition sites were identified using the NEBcutter v.2.0 (Vincze et al. 2003).

All of the nucleotide positions described in the text correspond to positions in the M. abscessus ATCC 19977 genome (GenBank accession NC_010397).

PRA-rpoB - For the PRA-rpoB determinations, the primers MycoF (Adékambi et al. 2003) and MycoR2 (Table II) were used to amplify a $221 \mathrm{bp}$ fragment of the rpo $B$ gene. The $15 \mu \mathrm{L}$ reactions were composed of the following reagents: $1.5 \mu \mathrm{L}$ of $10 \mathrm{X}$ Buffer $(150 \mathrm{mM}$ Tris$\mathrm{HCl}, \mathrm{pH} 8.75,500 \mathrm{mM} \mathrm{KCl}, 1 \%$ Triton X-100 and $20 \mathrm{mM}$ of $\mathrm{MgCl}_{2}$ ), $140 \mu \mathrm{M}$ of each dNTP, $0.4 \mu \mathrm{M}$ of each primer, $0.3 \mathrm{U}$ of Taq DNA Polymerase (RBC Biosciences Corp, Taipei, Taiwan) and $2 \mu \mathrm{L}$ of DNA. The PCR was performed using the following conditions: an initial denaturation step at $95^{\circ} \mathrm{C}$ for $3 \mathrm{~min}, 35$ cycles of $95^{\circ} \mathrm{C}$ for 30 $\mathrm{sec}, 65^{\circ} \mathrm{C}$ for $30 \mathrm{sec}$ and $72^{\circ} \mathrm{C}$ for $1 \mathrm{~min}$ and a final extension at $72^{\circ} \mathrm{C}$ for $5 \mathrm{~min}$. The digestion of $10 \mu \mathrm{L}$ aliquots of the PCR product using DpnII (New England Biolabs, Ipswich, MA, USA) was carried out in a final volume of $20 \mu \mathrm{L}$ according to the manufacturer's instructions.

PRA-16S - For the PRA-16S determinations, the primers 800F (Adékambi \& Drancourt 2004) and 16R1492 (Gomila et al. 2007) were used to amplify a 733 bp fragment. The same reagents, volumes and amplification conditions described above were used in the 16S rDNA PCR with the exception of changes in the $\mathrm{MgCl}_{2}$ concentration (1.5 $\mathrm{mM}$ final concentration) and the annealing temperature $\left(60^{\circ} \mathrm{C}\right)$. A $10 \mu \mathrm{L}$ aliquot of the PCR product was digested with XbaI (Promega, Madison, WI, USA) in a $20 \mu \mathrm{L}$ reaction according to the manufacturer's instructions.

gyrB sequence analysis - For the gyrB sequence analysis, a $524 \mathrm{bp}$ fragment was amplified using the primers 
TABLE I

Mycobacterium abscessus type 2 isolates used in phases Ia, Ib, IIa and IIb and results obtained with polymerase chain reaction restriction-enzyme analysis (PRA)-rpoB, PRA-16S and gyrB sequence analysis

\begin{tabular}{|c|c|c|c|c|c|c|}
\hline Phase & Isolate $^{a}$ & Year of isolation & State & PRA-rpoB & PRA-16S & gyrB analysis \\
\hline \multirow[t]{10}{*}{ Ia } & CCUG 48898 & & & - & - & - \\
\hline & CCUG 50184 & & & - & - & - \\
\hline & INCQS $00594^{b, c}$ & 2005 & PA & + & + & + \\
\hline & $\mathrm{B} 67^{d}$ & 2004 & PA & - & - & - \\
\hline & IAL $002^{e}$ & 2005 & $\mathrm{CE}$ & - & - & - \\
\hline & IAL $014^{e}$ & 2006 & SP & - & - & - \\
\hline & IAL $028^{e}$ & 2008 & SP & - & - & - \\
\hline & $\mathrm{P} 7^{e}$ & 2006 & PA & - & - & - \\
\hline & $\mathrm{P} 13^{e}$ & 2006 & PA & - & - & - \\
\hline & $\mathrm{P} 53^{e}$ & 2007 & PA & - & - & - \\
\hline \multirow[t]{47}{*}{$\mathrm{Ib}$} & $\mathrm{B} 52^{b, c}$ & 2004 & PA & + & + & + \\
\hline & CRM $0019^{b, c}$ & 2006 & RJ & + & + & + \\
\hline & CRM $0184^{b, c}$ & 2006 & RJ & + & + & + \\
\hline & RS $700345^{b, c}$ & 2007 & $\mathrm{RS}$ & + & + & + \\
\hline & $307597^{b, c}$ & 2007 & ES & + & + & + \\
\hline & IAL $040^{b, c}$ & 2008 & PR & + & + & + \\
\hline & IAL $041^{b, c}$ & 2008 & PR & + & + & + \\
\hline & IAL $042^{b, c}$ & 2008 & SP & + & + & + \\
\hline & IAL $043^{b, c}$ & 2008 & SP & + & + & + \\
\hline & CRM $270^{e, f}$ & 2007 & RJ & + & + & - \\
\hline & CRM $273^{e, f}$ & 2007 & $\mathrm{RJ}$ & + & + & - \\
\hline & IAL $020^{c, e}$ & 2006 & SP & + & + & + \\
\hline & IAL $034^{c, g}$ & 2006 & SP & + & + & + \\
\hline & IAL $036^{c, h}$ & 2006 & $\mathrm{CE}$ & + & + & - \\
\hline & $\mathrm{P} 10^{c, e}$ & 2005 & PA & + & + & - \\
\hline & IAL $001^{e}$ & 2005 & $\mathrm{CE}$ & - & - & - \\
\hline & IAL $003^{e}$ & 2005 & $\mathrm{CE}$ & - & + & - \\
\hline & IAL $004^{e}$ & 2006 & $\mathrm{CE}$ & - & - & - \\
\hline & IAL $005^{e}$ & 2006 & $\mathrm{CE}$ & - & - & - \\
\hline & IAL $006^{e}$ & 2006 & $\mathrm{CE}$ & - & - & - \\
\hline & IAL $007^{e}$ & 2008 & DF & - & - & - \\
\hline & IAL $008^{e}$ & 2007 & MS & - & - & - \\
\hline & IAL $009^{e}$ & 2006 & MS & - & - & - \\
\hline & IAL $010^{e}$ & 2007 & MT & - & - & - \\
\hline & IAL $011^{e}$ & 2007 & PI & - & - & - \\
\hline & IAL $012^{e}$ & 2005 & SP & - & - & - \\
\hline & IAL $013^{e}$ & 2005 & SP & - & - & - \\
\hline & IAL $015^{e}$ & 2006 & SP & - & - & - \\
\hline & IAL $016^{e}$ & 2007 & SP & - & - & - \\
\hline & IAL $017^{e}$ & 2007 & SP & - & - & - \\
\hline & IAL $018^{e}$ & 2007 & SP & - & - & - \\
\hline & IAL $019^{e}$ & 2007 & SP & - & - & - \\
\hline & IAL $021^{e}$ & 2006 & SP & - & - & - \\
\hline & IAL $024^{e}$ & 2005 & SP & - & - & - \\
\hline & IAL $029^{e}$ & 2005 & SP & - & - & - \\
\hline & IAL $035^{i}$ & 2007 & SP & - & - & - \\
\hline & IAL $037^{j}$ & 2006 & SP & - & - & - \\
\hline & MG3-6 ${ }^{k}$ & 2005 & ES & - & - & - \\
\hline & $\mathrm{P} 3^{e}$ & 2004 & PA & - & - & - \\
\hline & $\mathrm{P} 4^{e}$ & 2007 & PA & - & - & - \\
\hline & $\mathrm{P} 5^{e}$ & 2005 & PA & - & - & - \\
\hline & $\mathrm{P} 6^{e}$ & 2004 & PA & - & - & - \\
\hline & $\mathrm{P}^{e}$ & 2006 & PA & - & - & - \\
\hline & $\mathrm{P} 14^{e}$ & 2004 & PA & - & + & - \\
\hline & $\mathrm{P} 51^{e}$ & 2002 & PA & - & - & - \\
\hline & $\mathrm{P} 52^{e}$ & 2003 & PA & - & - & - \\
\hline & $\mathrm{P} 54^{e}$ & 2006 & PA & - & - & - \\
\hline
\end{tabular}




\begin{tabular}{|c|c|c|c|c|c|c|}
\hline Phase & Isolate $^{a}$ & Year of isolation & State & PRA-rpoB & PRA-16S & gyrB analysis \\
\hline \multirow[t]{15}{*}{ IIa } & CRM $311^{b, c}$ & 2008 & RJ & + & + & + \\
\hline & CRM $312^{b, c}$ & 2008 & RJ & + & + & + \\
\hline & CRM $313^{b, c}$ & 2008 & $\mathrm{RJ}$ & + & + & + \\
\hline & CRM $353^{b, c}$ & 2008 & PR & + & + & + \\
\hline & CRM $375^{e}, \mathrm{CRM}_{378^{e}}, \mathrm{CRM} 405^{e}$ & 2008 & $\mathrm{RJ}$ & - & - & - \\
\hline & CRM $409^{e,}$ CRM $416^{e}$ & 2008 & $\mathrm{RJ}$ & - & - & - \\
\hline & $\mathrm{CRM} 473^{e}, \mathrm{CRM} 639^{e}$ & 2009 & $\mathrm{RJ}$ & - & - & - \\
\hline & CRM $512^{e}$, CRM $640^{e}$ & 2009 & RJ & - & - & - \\
\hline & CRM $552^{c, g}$ & 2009 & $\mathrm{RJ}$ & + & + & + \\
\hline & CRM $572^{b, c}$ & 2009 & RJ & + & + & + \\
\hline & CRM $573^{e}$ & 2009 & RJ & - & + & - \\
\hline & CRM $575^{b, c}$ & 2009 & RJ & + & + & + \\
\hline & CRM $619^{b, c}$ & 2009 & PR & + & + & + \\
\hline & CRM $622^{c, g}$ & 2009 & $\mathrm{RJ}$ & + & + & + \\
\hline & CRM $642^{e}$ & 2009 & RJ & - & - & - \\
\hline \multirow[t]{26}{*}{$\mathrm{IIb}$} & $\begin{array}{l}\text { CRPHF } 3451^{e}, \text { CRPHF } 3498^{e}, \text { CRPHF } 4163^{e}, \\
\text { CRPHF } 4402^{e}, \text { CRPHF } 4406^{e}, \text { CRPHF } 4407^{e}\end{array}$ & 2009 & $\mathrm{RJ}$ & - & - & - \\
\hline & CRPHF $3452^{e}$, CRPHF $4343^{e}$ & 2009 & RJ & - & - & - \\
\hline & CRPHF $3464^{b, c}$ & 2009 & $\mathrm{RJ}$ & + & + & + \\
\hline & CRPHF $3541^{e}$, CRPHF $4811^{e}$ & 2009 & $\mathrm{PE}$ & - & - & - \\
\hline & CRPHF $3633^{e}$ & 2009 & $\mathrm{PE}$ & - & - & - \\
\hline & CRPHF $3634^{g}$ & 2009 & $\mathrm{PE}$ & - & - & - \\
\hline & CRPHF $3761^{c, g}$ & 2009 & $\mathrm{AL}$ & + & + & + \\
\hline & CRPHF $3850^{e}$ & 2009 & $\mathrm{PE}$ & - & - & - \\
\hline & CRPHF $3851^{b}$ & 2009 & $\mathrm{PE}$ & - & - & - \\
\hline & CRPHF $3882^{e}$ & 2009 & $\mathrm{RJ}$ & - & - & - \\
\hline & CRPHF $3918^{g}$ & 2009 & $\mathrm{RS}$ & - & - & - \\
\hline & CRPHF $3932^{g}$ & 2009 & $\mathrm{RS}$ & - & - & - \\
\hline & CRPHF $3941^{e}$ & 2009 & $\mathrm{RS}$ & - & - & - \\
\hline & CRPHF $4013^{e}$ & 2009 & MT & - & - & - \\
\hline & CRPHF $4168^{e}$ & 2009 & $\mathrm{RJ}$ & - & - & - \\
\hline & CRPHF $4301^{e}$ & 2009 & MA & - & - & - \\
\hline & CRPHF $4636^{b, c}$ & 2009 & RJ & + & + & + \\
\hline & CRPHF $4760^{e}$ & 2009 & $\mathrm{CE}$ & + & - & - \\
\hline & CRPHF $4817^{b}$ & 2009 & $\mathrm{PE}$ & - & - & - \\
\hline & $\begin{array}{l}\text { CRPHF } 5813^{e}, \text { CRPHF } 5814^{e}, \\
\text { CRPHF } 6107^{i}, \text { CRPHF } 6108^{i}\end{array}$ & 2009 & $\mathrm{GO}$ & - & - & - \\
\hline & CRPHF $6026^{e, f}$ CRPHF $6027^{e, f}$ & 2009 & RR & + & + & - \\
\hline & CRPHF $6244^{e}$ & 2010 & MS & - & - & - \\
\hline & CRPHF $6263^{d}$ & 2010 & $\mathrm{RO}$ & - & - & - \\
\hline & CRPHF $6492^{e, f}$, CRPHF $6493^{e, f}$ & 2010 & RS & + & + & - \\
\hline & CRPHF $6619^{e}$, CRPHF $6620^{e}$ & 2010 & RO & - & - & - \\
\hline & CRPHF $6979^{b}$ & 2010 & MG & - & - & - \\
\hline
\end{tabular}

$a$ : isolates obtained from the same patient were grouped; $b$ : surgical isolate; $c$ : isolates that belong to the epidemic strain; $d$ : injection site abscess; $e$ : sputum; $f$ : isolates genetically related to the epidemic strain; $g$ : bronchoalveolar lavage; $h$ : urine; $i$ : blood; $j$ : parotid nodule; $k$ : sewage; AL: Alagoas; CE: Ceará; DF: Federal District; ES: Espírito Santo; GO: Goiás; MA: Maranhão; MG: Minas Gerais; MS: Mato Grosso do Sul; MT: Mato Grosso; PA: Pará; PE: Pernambuco; PI: Piauí; PR: Paraná; RJ: Rio de Janeiro; RO: Rondônia; RR: Roraima; RS: Rio Grande do Sul; SP: São Paulo; +: epidemic strain by the test; -: different strain by the test.

GYRB7 and GYRB8 (Table II) in a $25 \mu \mathrm{L}$ reaction using the same reagents and concentrations described for PCR-rpoB but with a $60^{\circ} \mathrm{C}$ annealing temperature. The primer GYRB7 was used for sequencing.

Nucleotide sequence accessions - The sequences obtained from this work were deposited in GenBank under the following accessions: GU131159-GU131161, GU143881, GU143882,GU143885-GU143887,GU143888.2,GU143891, JQ582469-JQ582622 and JQ678790-JQ678815.

\section{RESULTS}

The development and evaluation of the tests for the rapid detection of the epidemic strain were performed in four consecutive phases (Ia, Ib, IIa and IIb) (Table I).

In phase Ia, the sequences of three genes ( $r p o B$, the 3 ' region of the 16S rDNA and $g y r B$ ) were analysed. The INCQS 00594 sequences were aligned to the corresponding sequences from $M$. bolletii CCUG 50184, M. massiliense CCUG 48898 and seven randomly se- 


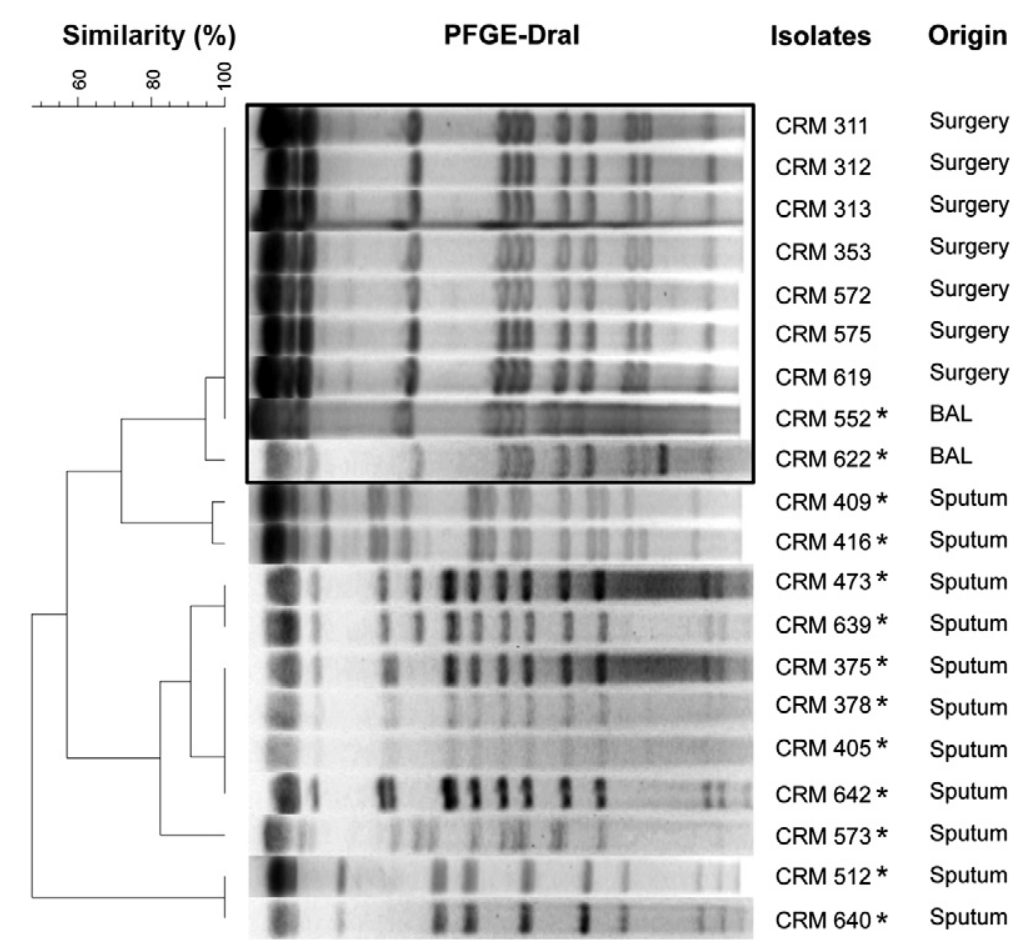

Fig. 1: pulsed field gel electrophoresis (PFGE)-DraI patterns of isolates from Federal University of Rio de Janeiro used in evaluation phase IIa. Nine isolates belonging to the epidemic strain are boxed. BAL: bronchoalveolar lavage. Asterisks mean Matsumoto et al. (2011).

lected isolates that did not belong to the epidemic strain (B67, IAL 002, IAL 014, IAL 028, P7, P13 and P53). The seven non-epidemic strains were not isolated from surgical-site infections and their rpoB sequences and PFGE patterns were different from those of the epidemic strain. All of the isolates showed the M. abscessus type 2 PRA-hsp 65 pattern.

The following SNPs that occur only in INCQS 00594 and are located in restriction enzyme recognition sites were identified: an A/G SNP at position 3,916,702 in $r p o B$, located in a DpnII restriction site (AATC in the epidemic strain and GATC in isolates from other strains), a T/C SNP at position 1,463,374 in the $16 \mathrm{~S}$ rDNA, located in a XbaI recognition site (TCTAGA in isolates belonging to the epidemic strain and CCTAGA in isolates that do not belong to the epidemic strain), and an A/C SNP at position 8,148 in $g y r B$, located in a TauI recognition site (GCSGC, where S indicates $\mathrm{C}$ or G; GAGGC in the epidemic strain; GCGGC in non-epidemic strain).

Three tests were developed in phase Ib, two tests are based on PRA (PRA-rpoB and PRA-16S) and one test is based on $g y r B$ sequencing.

The 752 bp rрoB fragment described by Adékambi et al. (2003) contains multiple DpnII recognition sites and a new primer, MycoR2 (Table II), was designed for the amplification of a $221 \mathrm{bp}$ fragment that contains only the DpnII site of interest. This DpnII restriction site is absent in the epidemic strain due to an $\mathrm{A} / \mathrm{G}$ substitution and the corresponding amplicon was not cut with this enzyme. In contrast, this DpnII restriction site was present in the analysed isolates that are not related to the epidemic strain and digestion of the PCR products from these isolates with DpnII generated two fragments of $168 \mathrm{bp}$ and $53 \mathrm{bp}$.

For the PRA-16S determination, primers $800 \mathrm{~F}$ and 16R1492 (Table II) were used to amplify a 733 bp fragment of the 16S rDNA gene and the amplicon was digested with XbaI. The XbaI restriction site was present exclusively in the strain INCQS 00594 and two fragments of $503 \mathrm{bp}$ and $230 \mathrm{bp}$ were produced by the PRA-16S test. The $16 \mathrm{~S}$ amplicons of the strains unrelated to the epidemic strain were not digested.

Additional TauI sites in $g y r B$ are present 20 bp downstream from the SNP of interest, thereby complicating the design of a PRA test. The relatively small size difference between the digested and non-digested fragments would not be detectable using agarose gels. As an alternative approach, the outbreak strain was discriminated using sequence analysis.

Of the 47 isolates used in the three tests in phase Ib, 13 belonged to the epidemic strain, nine were retrieved from surgical-site infections (307597, B52, CRM 0019, CRM 0184, IAL 040, IAL 041, IAL 042, IAL 043 and RS 700345) and four were isolated from other specimens (IAL 020 and P10 from sputum, IAL 034 from BAL and IAL 036 from urine). Two of the sputum isolates (CRM 270 and CRM 273) showed PFGE patterns that differed in two bands when compared to the patterns of the epidemic strain isolates and were thus considered genetically related to the epidemic strain (Matsumoto et al. 2011). The nine isolates from surgical-site infections and isolates IAL 020 and IAL 034 from the other specimens were correctly identified by the three 


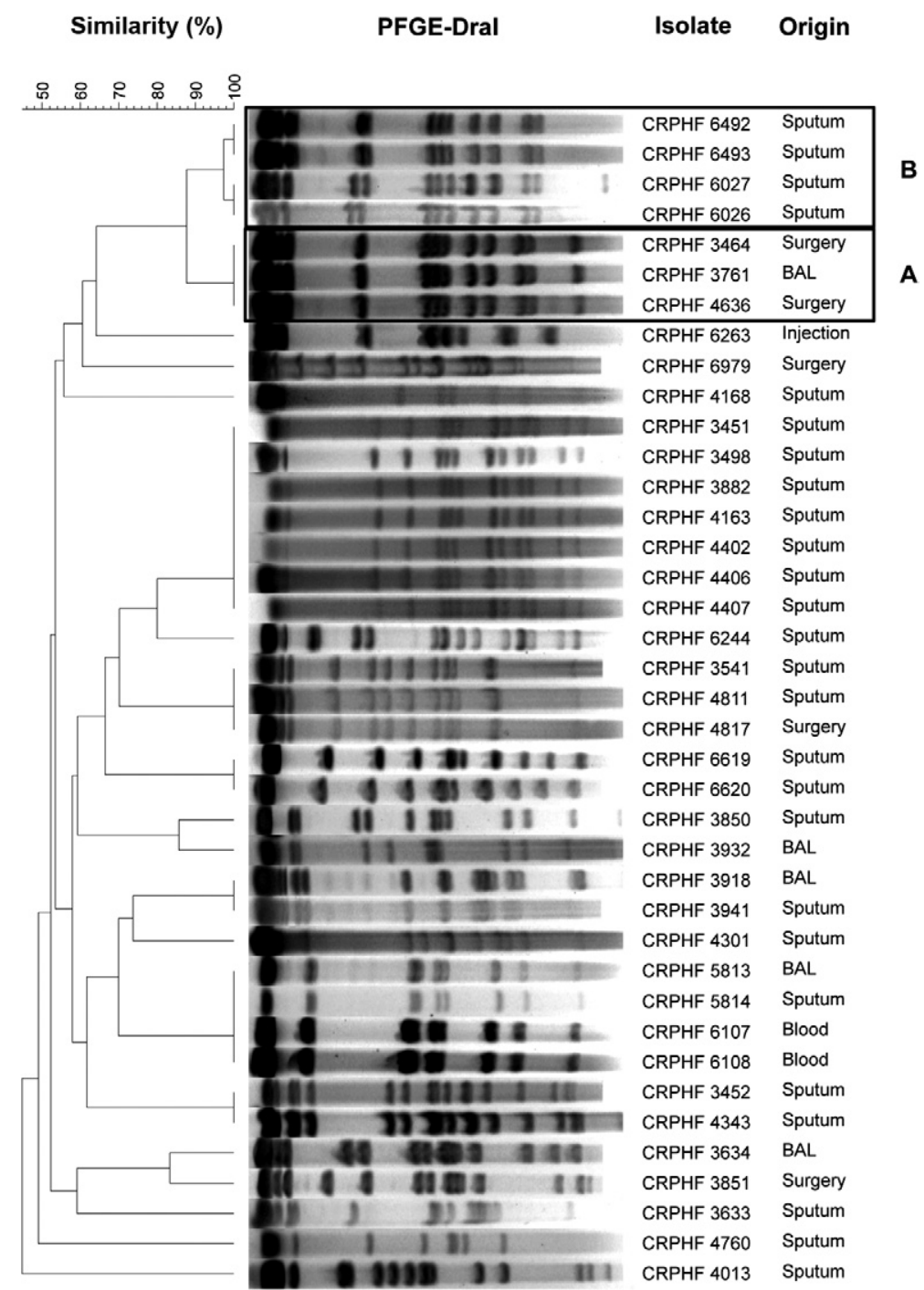

Fig. 2: pulsed field gel electrophoresis (PFGE)-DraI patterns of isolates from National Reference Laboratory Professor Hélio Fraga (CRPHF), state of Rio de Janeiro, used in evaluation phase IIb. A: isolates belonging to the epidemic strain; B: genetically related isolates; BAL: bronchoalveolar lavage.

tests as belonging to the epidemic strain. Two isolates that are genetically related to the epidemic strain (CRM 270 and CRM 273) tested positive by PRA-rpoB and PRA-16S, but did not show the SNP that is characteristic of the epidemic strain according to $g y r B$ sequence analysis. Two isolates from strains other than the epidemic strain (IAL 003 and P14) were identified by PRA-16S as belonging to the epidemic strain (i.e., false positives). False negative results from the $\operatorname{gyr} B$ sequence analysis were observed for isolates IAL 036 and P10, which belong to the epidemic strain (Table I). PRA-rpoB was selected for the evaluation phases IIa and IIb. Additionally, PRA-16S and $\operatorname{gyr} B$ sequence analyses were performed to complement the previously obtained results.

A total of 20 coded isolates from 15 patients were used in a blind evaluation of the PRA-rpoB test (phase IIa). Nine isolates showed positive results and were re- sistant to digestion with DpnII, which is characteristic of the epidemic strain. Seven of these isolates (CRM 311, CRM 312, CRM 313, CRM 353, CRM 572, CRM 575 and CRM 619) were obtained from surgical-site infections and two were isolated from BAL (CRM 552 and CRM 622). Eleven isolates, none of which were from surgical patients, tested negative (i.e., the rpoB amplicon was cut with DpnII). The results obtained from the $g y r B$ sequence analysis were consistent with the PRA-rpoB results. The PRA-16S test identified an additional isolate from a sputum sample (CRM 573) as belonging to the epidemic strain. The characterisation of these isolates by PFGE confirmed that the nine isolates that tested positive by the three developed tests belonged to the epidemic strain and that CRM 573 was a false positive result of PRA-16S (Fig. 1, Table I).

In the final evaluation (phase IIb), the PRA-rpoB determinations were performed in a different laboratory. 
TABLE II

Primers used for polymerase chain reaction and sequencing

\begin{tabular}{|c|c|c|c|c|}
\hline Gene & Primer & Sequence 5'-3' & $\begin{array}{c}\text { Temperature } \\
\left({ }^{\circ} \mathrm{C}\right)\end{array}$ & Reference \\
\hline \multirow[t]{3}{*}{ гров } & MycoF & GGCAAGGTCACCCCGAAGGG & 68 & Adékambi et al. (2003) \\
\hline & MycoR & AGCGGCTGCTGGGTGATCATC & 68 & \\
\hline & MycoR2 & CGCTTCTGCGCCACGTACA & 62 & This paper \\
\hline \multirow[t]{2}{*}{ 16S rDNA } & 16R1492 & TACGGCTACCTTGTTACGACTT $^{a}$ & 64 & Gomila et al. (2007) \\
\hline & $800 \mathrm{~F}$ & ATTAGATACCCTGGTAG & 48 & Adékambi and Drancourt (2004) \\
\hline \multirow[t]{8}{*}{ gyrB } & GYRB1 & AGAAGAAGAGTGCCAAGAGCG & 64 & This paper \\
\hline & GYRB2 & CTCATCGGTGAGCTTGATGGT & 64 & \\
\hline & GYRB3 & GACCAAGAAGACGGGTACCAC & 66 & \\
\hline & GYRB4 & GCTTGGTCTTGGTCTGGCCTT & 66 & \\
\hline & GYRB5 & GCCAAGGAGAAGAAGCTCCTC & 66 & \\
\hline & GYRB6 & CCATCAACACGATCTTGTGGT & 62 & \\
\hline & GYRB7 & GGGTTTTGAAGAACACCGAAG & 62 & \\
\hline & GYRB8 & GTCCTTGGCGTTTCGGGTGAT & 66 & \\
\hline
\end{tabular}

a: primer was modified according to the sequence of 16S rDNA in Mycobacterium abscessus ATCC 19977 (GenBank accession NC 010397).

The PRA-rpoB protocol and primers were sent to the laboratory at CRPHF and 39 isolates from 26 patients with the M. abscessus type 2 PRA-hsp 65 pattern were tested at CRPHF. Eight isolates from six patients tested positive by PRA-rpoB. Of these eight isolates, only two isolates (CRPHF 3464 and CRPHF 4636) were recovered from patients that had undergone surgical procedures. Five isolates (CRPHF 4760, CRPHF 6026, CRPHF 6027, CRPHF 6492 and CRPHF 6493) from three patients were obtained from sputum samples and one isolate (CRPHF 3761) was obtained from BAL. The remaining 31 isolates tested negative for the epidemic strain (Table I). These results were compared to the results obtained from the PRA-16S and $g y r B$ sequence analyses. All but one (CRPHF 4760) of the PRA-16S results were consistent with the results obtained by PRA$r p o B$. The gyrB SNP that is characteristic of the epidemic strain was detected only in the two surgical isolates and in the BAL isolate that tested positive by PRA-rpoB and PRA-16S. The comparison of these results with the results of the $r p o B$ sequencing and PFGE-DraI determinations showed that the two surgery isolates and the BAL isolate that tested positive according to all three of the developed tests (CRPHF 3464, CRPHF 3761 and CRPHF 4636) belonged to the epidemic strain and four out of five of the sputum isolates that tested positive by PRA-rpoB and PRA-16S (CRPHF 6026, CRPHF 6027, CRPHF 6492 and CRPHF 6493) were genetically related to the epidemic strain (Fig. 2). The fifth sputum isolate (CRPHF 4760) was not related to the epidemic strain and therefore was a false positive PRA-rpoB result. All of the remaining isolates, including three isolates from surgical-site infections (CRPHF 3851, CRPHF 4817 and CRPHF 6979), tested negative according to the three developed tests and belonged to strains other than the epidemic strain (Fig. 2, Table I).
Of the 116 isolates included in this study, 95 isolates corresponded to one isolate per patient, one environmental isolate and the $M$. bolletii and $M$. massiliense type strains. Of these 98 isolates, 26 belonged to the epidemic strain and 72 belonged to other strains (including the isolates that are genetically related to the epidemic strain). The PRA-rpoB test showed 100\% (26/26) sensitivity, i.e., all isolates belonging to the epidemic strain were correctly differentiated. The specificity of this test was $93.06 \%(67 / 72)$ due to five false positive results. The respective sensitivity values for the PRA-16S and gyrB sequence analyses were 100\% (26/26) and 92.31\% $(24 / 26)$ and the specificity values were $90.28 \%(65 / 72)$ and $100 \%(72 / 72)$, respectively (Table III).

\section{TABLE III}

Sensitivity and specificity of polymerase chain reaction restriction-enzyme analysis (PRA)-rpoB, PRA-16S and $\operatorname{gyr} B$ analysis of 98 isolates

\begin{tabular}{lccccc}
\hline \multirow{2}{*}{ Tests } & & \multicolumn{2}{c}{ PFGE } & Sensitivity \\
\cline { 2 - 6 } & & $\mathrm{P}$ & $\mathrm{N}$ & $\begin{array}{c}\text { Specificity } \\
(\%)\end{array}$ \\
\hline PRA-rpoB & $\mathrm{P}$ & 26 & 5 & 100 & 93.06 \\
PRA-16S & $\mathrm{N}$ & 0 & 67 & - & - \\
& $\mathrm{P}$ & 26 & 7 & 100 & 90.28 \\
gyrB analysis & $\mathrm{N}$ & 0 & 65 & - & - \\
& $\mathrm{P}$ & 24 & 0 & 92.31 & 100 \\
& $\mathrm{~N}$ & 2 & 72 & - & - \\
\hline
\end{tabular}

pulsed field gel electrophoresis (PFGE) was considered the gold standard method to discriminate the epidemic strain. N: different strain; P: epidemic strain. 


\section{DISCUSSION}

In this study, we developed three tests for the rapid discrimination of the epidemic strain among isolates previously identified as $M$. abscessus type 2 according to PRA-hsp65 assays.

Initially, the sequences of 15 DNA different regions that are conserved in mycobacteria were studied: $h s p 65$, rpoB, gyrA, gyrB, the 5' and 3' regions of the $16 \mathrm{~S}$ rDNA, $23 \mathrm{~S}$ rDNA, the 16S-23S internal transcribed sequence and genes previously used in multilocus sequence analysis (Macheras et al. 2011), argH, cya, glpK, gnd, pgm, pta and $p u r H$ (data not shown). The sequences of these genes from M. massiliense and M. bolletii were retrieved from GenBank and aligned to the corresponding sequences of INCQS 00594. Additionally, certain isolates included in phases Ia and Ib were sequenced. SNPs and enzyme restriction sites specific to the epidemic strain were identified in silico after sequence alignment. This paper describes the analysis of three DNA regions, $r p o B$, the 3' region of $16 \mathrm{~S}$ rDNA and $g y r B$, that contained SNPs that were exclusive to the epidemic strain and were located in enzyme recognition sites. The other studied regions, isolates, accessions and primers are provided in the supplementary data. The in silico analysis of $g l p K$ and pta showed that these genes could represent additional targets for the differentiation of the epidemic strain, although they were not used in further studies.

An A/G SNP at nucleotide position 3,916,702 corresponds to the first base of the 857th amino acid (Leu) of $r p o B$ and is a synonymous mutation. This SNP is one of two SNPs found in the rpoB sequence from INCQS 00594 (GenBank accession EU117207) that define the epidemic strain sequevar. An A/C SNP at nucleotide position 8,148 corresponds to the first base of the 634th gyrB codon (Arg) and is also a synonymous mutation. The T/C SNP at nucleotide position 1,463,374 detected in the 16S rDNA gene does not occur within hypervariable region $\mathrm{A}$ or $\mathrm{B}$, which are important for the discrimination of mycobacterial species (Tortoli 2003).

Of the 22 isolates obtained from surgical patients in this study, 19 epidemic strains and three non-epidemic strains (CRPHF 3851, CRPHF 4817 and CRPHF 6979) were correctly identified by the three methods.

Thirteen isolates from 11 patients (CRM 270, CRM 273, CRM 552, CRM 622, CRPHF 3761, CRPHF 6026, CRPHF 6027, CRPHF 6492, CRPHF 6493, IAL 020, IAL 034, IAL 036 and P10) were identified as belonging to the epidemic strain or genetically related according to the PFGE results, although these isolates were obtained from patients that were not submitted to surgical procedures. Interestingly, all of the genetically related isolates (CRM 270, CRM 273, CRPHF 6026, CRPHF 6027, CRPHF 6492 and CRPHF 6493) had rpoB sequences that were $100 \%$ similar to that of the epidemic strain INCQS 00594. In addition, all of these genetically related isolates were obtained from sputum, thereby indicating that a strain that is closely related to the epidemic strain can cause pulmonary disease. The 13 non-surgical isolates tested positive by PRA-rpoB and PRA-16S, although $8 / 13(61.54 \%)$ did not display the gyrB SNP that is characteristic of the epidemic strain.
During the development and evaluation of the PRArроB test, new surgical-site infections occurred in Manaus (AM) and Carazinho (RS) in 2010. Six isolates from $A M$ and eight from RS were screened using the PRA- $r р о B$ test and were identified as belonging to the epidemic strain. These results were corroborated by rpoB sequencing and PFGE analyses (data not shown). Therefore, PRA-rpoB proved useful for the rapid preliminary screening of the epidemic strain in field situations.

Using one isolate per patient (98 isolates, including 1 environmental isolate and the 2 type strains), we observed discordant results at frequencies of 5.10\% (5/98), 7.14\% (7/98) and 2.04\% (2/98) for the PRA-rpoB, PRA-16S and gyrB sequence analyses, respectively. The $100 \%$ sensitivity of the PRA-rрoB test was anticipated because it uses one of the two SNPs that are characteristic of the epidemic strain $r p o B$ sequevar. The sensitivity of the $\operatorname{gyr} B$ sequence analysis $(92.31 \%)$ was lower than the sensitivity values of the other two tests ( $100 \%$ each), although no false positive results were obtained in the $\operatorname{gyr} B$ test and the specificity $(100 \%)$ was higher than those of the PRA-rpoB and PRA$16 \mathrm{~S}$ tests. It is important to note that the PRA-rpoB and the PRA-16S tests, but not the $\operatorname{gyr} B$ sequence analysis, identified the genetically related isolates (6 isolates from 4 patients) as belonging to the outbreak strain. Therefore, the $\operatorname{gyr} B$ sequence analysis appears to be more specific to the identification of the outbreak strain, whereas the other two tests can also be used to identify genetically related isolates. The choice of specific tests could, therefore, depend on the objective of the analysis.

For 11 patients, multiple isolates from each patient were studied. The results of PRA-rpoB, PRA-16S and $\operatorname{gyr} B$ sequence analysis were consistent for all of the isolates from the same patient, confirming the stability of the three developed tests.

The PRA-rpo $B$ test proved to be an efficient tool for the differentiation of the epidemic strain. The PRA-16S and the $g y r B$ sequence analyses were shown to be useful as additional tests. The rapid tests developed here can be performed in one day and the interpretation is straightforward for both the PRA tests (i.e., the presence or absence of digestion) and sequence analysis (i.e., the detection of one SNP). In contrast, PFGE requires approximately two weeks for completion, including the necessary culture period. Moreover, PFGE requires technical training, expensive equipment and software for analysis if a large number of isolates are assayed. The PRA-rpoB test can be performed in a shorter amount of time than PFGE and is easily executed, especially in laboratories that already use the PRA-hsp 65 test for mycobacteria identification. The developed tests could be implemented for the epidemiological surveillance for the epidemic strain among surgical patient isolates that show the M. abscessus type 2 PRA-hsp 65 pattern.

\section{REFERENCES}

Adékambi T, Colson P, Drancourt M 2003. rpoB-based identification of nonpigmented and late-pigmenting rapidly growing mycobacteria. J Clin Microbiol 41: 5699-5708.

Adékambi T, Drancourt M 2004. Dissection of phylogenetic relationships among 19 rapidly growing Mycobacterium species by $16 \mathrm{~S}$ 
rRNA, hsp65, sodA, recA and rpoB gene sequencing. Int $J$ Syst Evol Microbiol 54: 2095-2105.

ANVISA - Agência Nacional de Vigilância Sanitária 2009. [homepage on the Internet]. Casos de infecção por micobactérias não tuberculosas notificadas [accessed in 2011 Dec 26]. Available from: anvisa.gov.br/hotsite/hotsite micobacteria/notificados.pdf.

ANVISA - Agência Nacional de Vigilância Sanitária 2011. [homepage on the Internet]. Relatório descrito de investigação de casos de infecções por micobactérias não tuberculosas de crescimento rápido (MCR) no Brasil no período de 1998 a 2009. [accessed in 2011 Dec 26]. Available from: anvisa.gov.br/hotsite/hotsite_micobacteria/relatorio_descrito_mcr_16_02_11.pdf.

Cardoso AM, Martins de Sousa E, Viana-Niero C, Bonfim de Bortoli F, Pereira das Neves ZC, Leão SC, Junqueira-Kipnis AP, Kipnis A 2008. Emergence of nosocomial Mycobacterium massiliense infection in Goiás, Brazil. Microbes Infect 10: 1552-1557.

da Costa AR, Lopes ML, Leão SC, Schneider MP, de Sousa MS, Suffys PN, Corvelo TC, Lima KV 2009. Molecular identification of rapidly growing mycobacteria isolates from pulmonary specimens of patients in the state of Pará, Amazon Region, Brazil. Diagn Microbiol Infect Dis 65: 358-364.

Devallois A, Goh KS, Rastogi N 1997. Rapid identification of mycobacteria to species level by PCR-restriction fragment length polymorphism analysis of the $h s p 65$ gene and proposition of an algorithm to differentiate 34 mycobacterial species. J Clin Microbiol 35: 2969-2973.

Duarte RS, Lourenço MC, Fonseca L de S, Leão SC, Amorim E de L, Rocha IL, Coelho FS, Viana-Niero C, Gomes KM, da Silva MG, Lorena NS, Pitombo MB, Ferreira RM, Garcia MH, de Oliveira GP, Lupi O, Vilaca BR, Serradas LR, Chebabo A, Marques EA, Teixeira LM, Dalcolmo M, Senna SG, Sampaio JL 2009. Epidemic of postsurgical infections caused by Mycobacterium massiliense. J Clin Microbiol 47: 2149-2155.

Gomila M, Ramirez A, Lalucat J 2007. Diversity of environmental mycobacterium isolates from hemodialysis water as shown by a multigene sequencing approach. Appl Environ Microbiol 73: 3787-3797.

Hall TA 1999. BioEdit: a user-friendly biological sequence alignment editor and analysis program for Windows 95/98/NT. Nucleic Acids Symp Ser 41: 95-98.

Leão SC, Tortoli E, Euzeby JP, Garcia MJ 2011. Proposal that Mycobacterium massiliense and Mycobacterium bolletii be united and reclassified as Mycobacterium abscessus subsp. bolletii comb. nov., designation of Mycobacterium abscessus subsp. abscessus subsp. nov. and emended description of Mycobacterium abscessus. Int J Syst Evol Microbiol 61: 2311-2313.

Leão SC, Tortoli E, Viana-Niero C, Ueki SY, Lima KV, Lopes ML, Yubero J, Menendez MC, Garcia MJ 2009. Characterization of mycobacteria from a major Brazilian outbreak suggests that revision of the taxonomic status of members of the Mycobacte- rium chelonae-M. abscessus group is needed. J Clin Microbiol 47: 2691-2698.

Leão SC, Viana-Niero C, Matsumoto CK, Lima KV, Lopes ML, Palaci M, Hadad DJ, Vinhas S, Duarte RS, Lourenço MC, Kipnis A, das Neves ZC, Gabardo BM, Ribeiro MO, Baethgen L, de Assis DB, Madalosso G, Chimara E, Dalcolmo MP 2010. Epidemic of surgical-site infections by a single clone of rapidly growing mycobacteria in Brazil. Future Microbiol 5: 971-980.

Macheras E, Roux AL, Bastian S, Leão SC, Palaci M, Sivadon-Tardy V, Gutierrez C, Richter E, Rusch-Gerdes S, Pfyffer G, Bodmer T, Cambau E, Gaillard JL, Heym B 2011. Multilocus sequence analysis and rpoB sequencing of Mycobacterium abscessus (sensu lato) strains. J Clin Microbiol 49: 491-499.

Macheras E, Roux AL, Ripoll F, Sivadon-Tardy V, Gutierrez C, Gaillard JL, Heym B 2009. Inaccuracy of single-target sequencing for discriminating species of the Mycobacterium abscessus group. J Clin Microbiol 47: 2596-2600.

Matsumoto CK, Chimara E, Bombarda S, Duarte RS, Leão SC 2011. Diversity of pulsed-field gel electrophoresis patterns of Mycobacterium abscessus type 2 clinical isolates. J Clin Microbiol 49: 62-68.

Roth A, Reischl U, Streubel A, Naumann L, Kroppenstedt RM, Habicht M, Fischer M, Mauch H 2000. Novel diagnostic algorithm for identification of mycobacteria using genus-specific amplification of the 16S-23S rRNA gene spacer and restriction endonucleases. J Clin Microbiol 38: 1094-1104.

Sampaio JL, Viana-Niero C, de Freitas D, Hofling-Lima AL, Leão SC 2006. Enterobacterial repetitive intergenic consensus PCR is a useful tool for typing Mycobacterium chelonae and Mycobacterium abscessus isolates. Diagn Microbiol Infect Dis 55: 107-118.

Thompson JD, Higgins DG, Gibson TJ 1994. CLUSTALW: improving the sensitivity of progressive multiple sequence alignment through sequence weighting, position-specific gap penalties and weight matrix choice. Nucleic acids research 22: 4673-4680.

Tortoli E 2003. Impact of genotypic studies on mycobacterial taxonomy: the new mycobacteria of the 1990s. Clin Microbiol Rev 16: $319-354$

van Belkum A, Tassios PT, Dijkshoorn L, Haeggman S, Cookson B, Fry NK, Fussing V, Green J, Feil E, Gerner-Smidt P, Brisse S, Struelens M 2007. Guidelines for the validation and application of typing methods for use in bacterial epidemiology. Clin Microbiol Infect 13 (Suppl. 3): 1-46.

Viana-Niero C, Lima KV, Lopes ML, da Silva Rabello MC, Marsola LR, Brilhante VC, Durham AM, Leão SC 2008. Molecular characterization of Mycobacterium massiliense and Mycobacterium bolletii in isolates collected from outbreaks of infections after laparoscopic surgeries and cosmetic procedures. $J$ Clin Microbiol 46: 850-855.

Vincze T, Posfai J, Roberts RJ 2003. NEBcutter: a program to cleave DNA with restriction enzymes. Nucleic Acids Res 31: 3688-3691. 
Twelve conserved DNA regions studied

\begin{tabular}{|c|c|c|c|c|c|c|c|c|c|c|c|c|}
\hline Isolates & $\begin{array}{c}\text { 5' region of } \\
\text { 16S rDNA } \\
(448 \mathrm{bp})\end{array}$ & $\begin{array}{l}\text { 23S rDNA } \\
(3,112 \mathrm{bp})\end{array}$ & $\begin{array}{c}\text { ITS } \\
(216 \mathrm{bp})\end{array}$ & $\begin{array}{c}g y r A \\
(549 \mathrm{bp})\end{array}$ & $\begin{array}{c}\text { hsp65 } \\
\text { (401 bp) }\end{array}$ & $\begin{array}{c}\operatorname{argH} \\
\text { (533 bp) }\end{array}$ & $\begin{array}{c}c y a \\
(513 \mathrm{bp})\end{array}$ & $\begin{array}{c}g l p K \\
(554 \mathrm{bp})\end{array}$ & $\begin{array}{c}\text { gnd } \\
\text { (494 bp) }\end{array}$ & $\begin{array}{c}p g m \\
\text { (559 bp) }\end{array}$ & $\begin{array}{c}p t a \\
(673 \mathrm{bp})\end{array}$ & $\begin{array}{c}\text { purH } \\
(598 \text { bp) }\end{array}$ \\
\hline INCQS 00594 & GU143891 ${ }^{a}$ & GU143886 & EU117206 & GU131159 & EU117205 & JQ582469 & JQ582470 & JQ582577 & JQ582471 & JQ582592 & JQ582600 & JQ582608 \\
\hline Mycobacterium bolletii & AY859681 & GU143888.2 & AM421291 & GU131161 & EU266576 & FJ609780 & FJ609784 & FJ609781 & FJ609783 & HM371394 & HM371395 & HM371396 \\
\hline Mycobacterium massiliense & AY593980 & GU143887 & AM421292 & GU131160 & EU266578 & FJ609785 & FJ609789 & FJ609786 & FJ609788 & HM371391 & HM371392 & HM371393 \\
\hline B $67^{b}$ & - & - & - & JQ582585 & EU220417 & - & - & JQ582578 & - & JQ582593 & JQ582601 & JQ582609 \\
\hline IAL $002^{c}$ & - & - & - & JQ582586 & - & - & - & JQ582579 & - & JQ582594 & JQ582602 & JQ582610 \\
\hline IAL $014^{c}$ & - & - & - & JQ582587 & - & - & - & JQ582580 & - & JQ582595 & JQ582603 & JQ582611 \\
\hline IAL $028^{c}$ & - & - & - & JQ582588 & - & - & - & JQ582581 & - & JQ582596 & JQ582604 & JQ582612 \\
\hline $\mathrm{P} 7^{d}$ & FJ590460 & - & - & JQ582589 & FJ536241 & - & - & JQ582582 & - & JQ582597 & JQ582605 & JQ582613 \\
\hline $\mathrm{P} 13^{d}$ & FJ590466 & - & - & JQ582590 & FJ536247 & - & - & JQ582583 & - & JQ582598 & JQ582606 & JQ582614 \\
\hline $\mathrm{P} 53^{d}$ & - & - & - & JQ582591 & - & - & - & JQ582584 & - & JQ582599 & JQ582607 & JQ582615 \\
\hline $\mathrm{P} 3^{d}$ & - & - & - & - & FJ536237 & - & - & - & - & - & - & - \\
\hline $\mathrm{P} 4^{d}$ & - & - & - & - & FJ536238 & - & - & - & - & - & - & - \\
\hline $\mathrm{P} 5^{d}$ & - & - & - & - & FJ536239 & - & - & - & - & - & - & - \\
\hline $\mathrm{P} 6^{d}$ & FJ590459 & - & - & - & FJ536240 & - & - & - & - & - & - & - \\
\hline $\mathrm{P}^{d}$ & FJ590461 & - & - & - & FJ536242 & - & - & - & - & - & - & - \\
\hline $\mathrm{P}^{d}$ & FJ590462 & - & - & - & FJ536243 & - & - & - & - & - & - & - \\
\hline $\mathrm{P} 10^{d}$ & FJ590463 & - & - & - & FJ536244 & - & - & - & - & - & - & - \\
\hline $\mathrm{P} 11^{d}$ & FJ590464 & - & - & - & FJ536245 & - & - & - & - & - & - & - \\
\hline $\mathrm{P} 12^{d}$ & FJ590465 & - & - & - & FJ536246 & - & - & - & - & - & - & - \\
\hline $\mathrm{P} 14^{d}$ & FJ590467 & - & - & - & FJ536248 & - & - & - & - & - & - & - \\
\hline $\mathrm{P} 15^{d}$ & FJ590468 & - & - & - & FJ536249 & - & - & - & - & - & - & - \\
\hline $\mathrm{B} 66^{b}$ & - & - & - & - & EU220418 & - & - & - & - & - & - & - \\
\hline $\mathrm{B} 62^{b}$ & - & - & - & - & EU220419 & - & - & - & - & - & - & - \\
\hline $\mathrm{B} 60^{b}$ & - & - & - & - & EU220420 & - & - & - & - & - & - & - \\
\hline
\end{tabular}

sequences were aligned using CLUSTALW Multiple Alignment program (Thompson et al. 1994) included in BioEdit package version 7.0.5.3 (Hall 1999) for identification of single nucleotide polymorphisms (SNPs) present exclusively in INCQS 00594. Sizes of the analyzed fragments are in parenthesis. Exclusive SNPs of INCQS 00594 were not found in 23S rDNA, $\operatorname{argH}, c y a$ and gnd and the internal transcribed sequence (ITS) region after alignment with two type strains. Alignment of the 5' region of $16 \mathrm{~S}$ rDNA and hsp65 sequences of INCQS 00594 and sequences available in GenBank has not evidenced exclusive SNPs for discrimination of the epidemic strain. $g l p K$ and $p t a$ could represent other targets to differentiate the epidemic strain. $a$ : GenBank accession; $b$ : Viana-Niero et al. (2008); $c$ : Matsumoto et al. (2011); $d$ : da Costa et al. (2009); -: not analyzed. 
Primers used for polymerase chain reaction and sequencing of conserved DNA regions

\begin{tabular}{|c|c|c|c|c|}
\hline Genes & Primers & Sequences 5'-3' & $\begin{array}{c}\text { Temperature } \\
\left({ }^{\circ} \mathrm{C}\right)\end{array}$ & References \\
\hline 23S rDNA & $\begin{array}{l}\text { Spl } \\
23 \mathrm{~S}-1 \mathrm{R} \\
23 \mathrm{~S}-2 \mathrm{~F} \\
23 \mathrm{~S}-2 \mathrm{R} \\
23 \mathrm{~S}-3 \mathrm{~F} \\
23 \mathrm{~S}-3 \mathrm{R} \\
23 \mathrm{~S}-4 \mathrm{~F} \\
23 \mathrm{~S}-4 \mathrm{R} \\
23 \mathrm{~S}-5 \mathrm{~F} \\
23 \mathrm{~S}-5 \mathrm{R} \\
23 \mathrm{~S}-6 \mathrm{~F} \\
23 \mathrm{~S}-6 \mathrm{R} \\
23 \mathrm{~S}-7 \mathrm{~F} \\
23 \mathrm{~S}-7 \mathrm{R} \\
23 \mathrm{~S}-8 \mathrm{R}\end{array}$ & $\begin{array}{c}\text { ACCTCCTTTCTAAGGAGCACC } \\
\text { CCCAAAGCCTATATATTCAGC } \\
\text { AGCCTCGGGGAGCTGCCAACC } \\
\text { GAGGCTTTGACGGATTGTAGG } \\
\text { ACTCTCTAGTGACCGATAGCG } \\
\text { AGTTTGGCTGACGTCAGTAAC } \\
\text { ATTTAGGTGCAGCGTCGCGTG } \\
\text { AAGTTTCTCACTTGCCTGTCG } \\
\text { AGGGGAGCGTCCTGCACCCAG } \\
\text { CGAAGTTACGGGGGTATTTTG } \\
\text { TATGCTGCCAAGAAAAGCCTC } \\
\text { TGTCCCTGGACCGGATATACG } \\
\text { TGTGAAGCAGGCACGCCAGTG } \\
\text { GTCCCGGTCCTCTCGTACTAG } \\
\text { GTGGAGTGTGGTCGTCAAATG }\end{array}$ & $\begin{array}{l}64 \\
60 \\
72 \\
64 \\
64 \\
62 \\
66 \\
62 \\
72 \\
62 \\
62 \\
66 \\
68 \\
68 \\
62\end{array}$ & $\begin{array}{l}\text { Roth et al. (2000) } \\
\text { This paper }\end{array}$ \\
\hline gyrA & $\begin{array}{l}\text { GyrA-F } \\
\text { GyrA-R }\end{array}$ & $\begin{array}{l}\text { GCCGTCGACCGCATCGAACCG } \\
\text { GTTGTCCAACGCCCAGTAGAC }\end{array}$ & $\begin{array}{l}72 \\
66\end{array}$ & This paper \\
\hline $\arg H$ & $\begin{array}{c}\text { ARGHF } \\
\text { ARGHSR1 }\end{array}$ & $\begin{array}{l}\text { GACGAGGGCGACAGCTTC } \\
\text { GTGCGCGAGCAGATGATG }\end{array}$ & $\begin{array}{l}60 \\
58\end{array}$ & Macheras et al. (2009) \\
\hline cya & $\begin{array}{l}\text { ACF } \\
\text { ACSR1 }\end{array}$ & $\begin{array}{l}\text { GTGAAGCGGGCCAAGAAG } \\
\text { AACTGGGAGGCCAGGAGC }\end{array}$ & $\begin{array}{l}58 \\
60\end{array}$ & Macheras et al. (2009) \\
\hline $\operatorname{glpK}$ & $\begin{array}{l}\text { GLPKSF1 } \\
\text { GLPKSFR2 }\end{array}$ & $\begin{array}{l}\text { AATCTCACCGGCGGTGTC } \\
\text { GGACAGACCCACGATGGC }\end{array}$ & $\begin{array}{l}58 \\
60\end{array}$ & Macheras et al. (2009) \\
\hline gnd & $\begin{array}{l}\text { GNDF } \\
\text { GNDSR1 }\end{array}$ & $\begin{array}{l}\text { GTGACGTCGGAGTGGTTGG } \\
\text { CTTCGCCTCAGGTCAGCTC }\end{array}$ & $\begin{array}{l}62 \\
62\end{array}$ & Macheras et al. (2009) \\
\hline pgm & $\begin{array}{l}\text { PGMSF1 } \\
\text { PGMSR2 }\end{array}$ & $\begin{array}{l}\text { CCATTTGAACCCGACCGG } \\
\text { GTGCCAACGAGATCCTGCG }\end{array}$ & $\begin{array}{l}58 \\
62\end{array}$ & Macheras et al. (2011) \\
\hline pta & $\begin{array}{l}\text { PTASF1 } \\
\text { PTASR2 }\end{array}$ & $\begin{array}{l}\text { GATCGGGCGTCATGCCCT } \\
\text { ACGAGGCACTGCTCTCCC }\end{array}$ & $\begin{array}{l}60 \\
60\end{array}$ & Macheras et al. (2011) \\
\hline purH & $\begin{array}{l}\text { PURHSF1 } \\
\text { PURHSR2 }\end{array}$ & $\begin{array}{l}\text { CGGAGGCTTCACCCTGGA } \\
\text { CAGGCCACCGCTGATCTG }\end{array}$ & $\begin{array}{l}60 \\
60\end{array}$ & Macheras et al. (2011) \\
\hline
\end{tabular}

